\title{
Synthesis and application of novel 4,5,6,7-tetrahydrobenzothiazole based azo disperse dyes
}

\author{
Samir J. Naik ${ }^{\dagger}$ and Uma P. Halkar* \\ Department of Chemistry, D.G.Ruparel College, Senapati Bapat Marg, Mahim, \\ Mumbai 400016, India \\ ${ }^{\dagger}$ Present Address: Process Research laboratory, Glenmark Research Centre, T.T.C. Industrial \\ Area, Mahape, Navi, Mumbai400709 India \\ E-mail: druphalkar@yahoo.com
}

(received 22 Nov 04; accepted 19 Apr 05; published on the web 21 Apr 05)

\begin{abstract}
3,3,5-Trimethylcyclohexanone was subjected to Hantzsch synthesis by treating with iodine and thiourea to give an intermediate 2-Amino-5,5,7-trimethyl-4,5,6,7-tetrahydrobenzothiazole. This was then diazotized and coupled with various $\mathrm{N}, \mathrm{N}$-dialkylaniline derivatives to give a series of novel disperse dyes. These dyes were characterized by spectral studies. The dyeing performance of these dyes was assessed on polyester fabric.
\end{abstract}

Keywords: 2-Aminothiazole, Hantzsch, azo dyes

\section{Introduction}

Heterocyclic amines have been used extensively in the preparation of disperse dyes. These dyes show outstanding discharge-ability on polyester. Disperse dyes before 1950 were mostly amino anthraquinone derivatives. Though these dyes are bright in color they have limitations of poor discharge-ability and are sensitive to the oxides of nitrogen. The derivatives of 2-aminothiazole are used as heterocyclic diazo components in disperse dyes. ${ }^{1}$ Dyes from 2-amino-5-nitrothiazole have been reported to have high extinction coefficient. They are reddish blue depending upon the substitution pattern in the coupler. ${ }^{2-9}$ Azo dyes of this type are classified as donor-acceptor chromogen. A red shift is observed by introduction of electron withdrawing substituent in the coupler. An interesting anomaly is the large bathochromic shift produced by m-acetamido group in the coupler. Further work carried out on 2-thiazolylazo dyes yielded violet dyes from 2amino-5-alkoxycarbonyl-4-trifluoromethylthiazole, ${ }^{10}$ from 5-alkylsulfonyl-2-aminothiazole, ${ }^{11}$ 5formyl-4-halothiazole ${ }^{12}$ and from 5-alkylsulfonyl-2-amino-5-nitrothiazole. ${ }^{13}$ James, Straley and David $^{14-15}$ have prepared blue azo dyes and greenish-blue azo dyes by coupling diazotized 2- 
aminothiazole derivatives with tetrahydroquinolone derivatives. Diazotization of 2aminobenzothiazole ${ }^{16}$ and coupling with $\mathrm{N}, \mathrm{N}$-dialkylated anilines has been reported to give red dyes. The whole range of mono and disubstituted derivatives of 2-aminobenzothiazole appear in important commercial red dyes. ${ }^{17}$ This prompted us to use a new substituted 2-amino-4,5,6,7tetrahydrobenzothiazole as a starting synthon for preparing dyes. Dyes with increased light fastness and sublimation fastness were prepared from aniline type coupling components containing one or more $\mathrm{N}$-alkyl groups substituted with groups such as alkoxy and cyano. ${ }^{18} \mathrm{~A}$ vast amount of work has been reported on aminothiazole based dyes ${ }^{19-26}$ in the last decade. Hantzsch $^{27}$ synthesis of 2-aminothiazole involves the condensation of thiourea and $\alpha$-haloketones or aldehydes to yield the corresponding 2-aminothiazole. We used a variation of Hantzsch aminothiazole synthesis ${ }^{28-29}$ for preparing the intermediate 2-amino-5,5,7-trimethyl-4,5,6,7tetrahydrobenzothiazole. This was then used as a starting material for preparing dyes by diazotization and coupling with various N,N-dialkylated aniline derivatives.
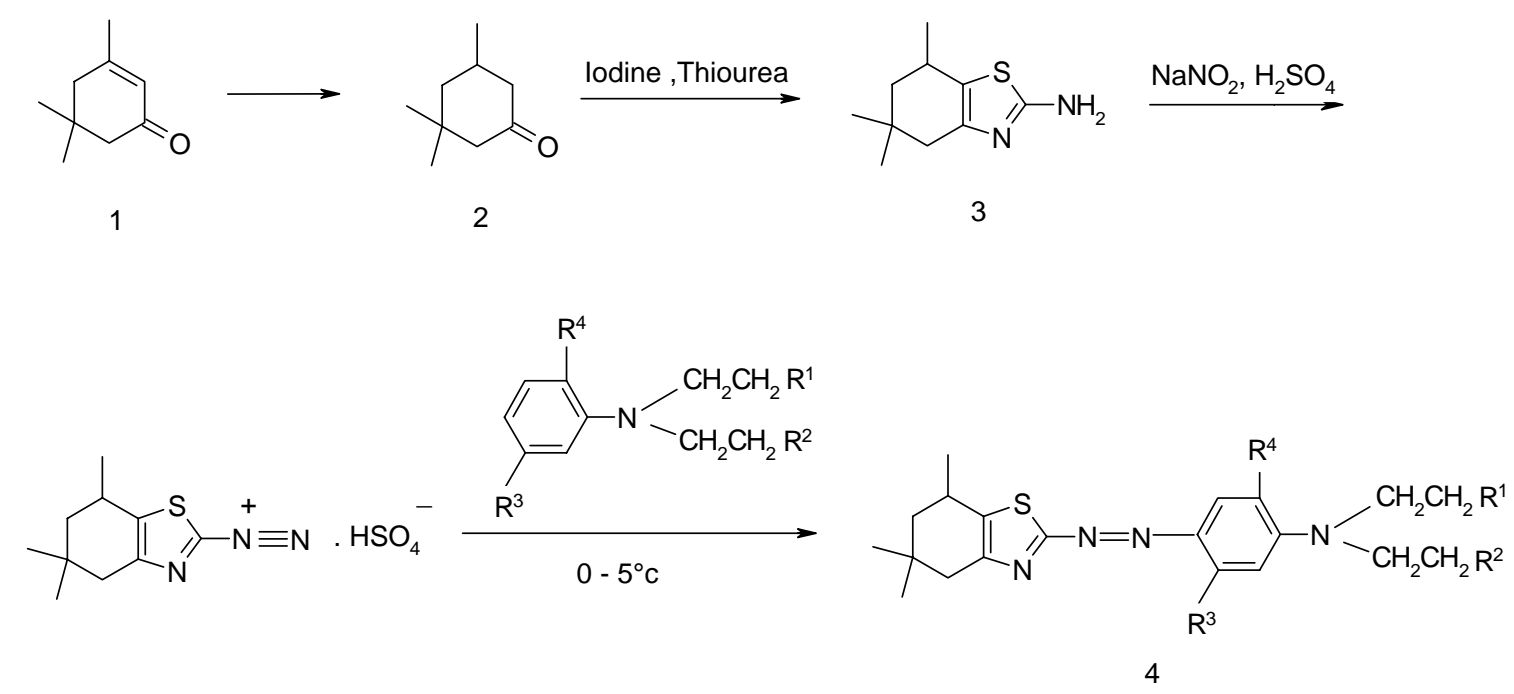

Figure 1

\section{Results and Discussion}

In the present study we report the synthesis of the azo dyes derived from a synthon 2-amino5,5,7-trimethyl-4,5,6,7-tetrahydrobenzothiazole (3). The intermediate 2-amino-5,5,7-trimethyl4,5,6,7-tetrahydrobenzothiazole (3) was synthesized from 3,3,5-trimethylcyclohexanone (2) using a variation of Hantzsch aminothiazole synthesis. 3,3,5-Trimethylcyclohexanone was treated with iodine and thiourea in alcoholic medium, wherein $\alpha$-iodination occurs at the $6^{\text {th }}$ position in 3,3,5-trimethylcyclohexanone followed by insitu condensation with thiourea. This then cylices with the elimination of $\mathrm{HI}$ to give 2-amino-5,5,7-trimethyl-4,5,6,7tetrahydrobenzothiazole (3). The presence of the gem dimethyl group at the $3^{\text {rd }}$ position and the 
use of a bulky iodine molecule as a halogenating agent prevented the halogenation of the $2^{\text {nd }}$ position in 3,3,5-trimethylcyclohexanone. Thus avoiding the formation of the other isomer 2amino-5,7,7-trimethyl-4,5,6,7-tetrahydrobenzothiazole. The compound (3) was characterized by elemental analysis. The I.R spectra of compound (3) showed the presence of a peak at $3329 \mathrm{~cm}^{-1}$ and $1362 \mathrm{~cm}^{-1}$ corresponding to the amino group and the gem dimethyl group respectively. I.R spectra also confirmed the absence of a peak at $1720 \mathrm{~cm}^{-1}$ (keto group), which was present in the starting material. Compound (3) was further confirmed by its ${ }^{1} \mathrm{H}$ NMR spectra recorded in $\mathrm{CDCl}_{3}$. Compound (3) was also confirmed by mass spectra which showed the molecular ion peak $\mathrm{m} / \mathrm{z}=197[\mathrm{~m}+\mathrm{H}]^{+}$, the molecular weight of the compound being 196.

Compound (3) was diazotized using nitrosyl sulfuric acid in a non-aqueous medium. The resulting diazonium salt solution was coupled with various $N, N$-dialkylaniline derivatives to obtain dyes. The dyes were purified by column chromatography and TLC determined the purity of the dyes. All the dyes (compounds 4a-4i) were characterized by their elemental analysis, I.R, ${ }^{1} \mathrm{H}$ NMR and mass spectroscopy. The infrared spectra of the dyes showed $\mathrm{C}-\mathrm{H}$ stretching vibrations of the aromatic ring appearing at 700-820 $\mathrm{cm}^{-1}$. The azo group stretching vibration band appeared at 1500-1530 $\mathrm{cm}^{-1}$, the stretching vibrations of alkyl group appeared at 2926-2960 $\mathrm{cm}^{-1}$. Dyes with acetylamino group at ortho position to the azo group showed N-H stretching vibration band at $3400-3500 \mathrm{~cm}^{-1}$. The ${ }^{1} \mathrm{H}$ NMR spectra confirmed the presence of the gem dimethyl group with a signal at $\delta 1.3-1.4(6 \mathrm{H})$.

\section{Visible absorption spectroscopic properties and dyeing properties of the dyes $4 a-4 i$}

The absorption maxima of the compounds 4a-4i recorded in their DMF solutions are shown in Table 1. Their absorption maxima were in the range of 480-532 $\mathrm{nm}$. The color of the dyes is affected by the substituents in the coupler constituent. The introduction of electron-donating or electron-withdrawing groups at suitable positions in the coupling components affects the absorption characteristics of the dyes. Bathochromic shift can be obtained by enhancing electron donor properties of the couplers. Dyes 4a-4i were applied on polyester fabric as $1 \%$ shade. $2 \mathrm{~g}$ of polyester fabric was used for dyeing and a laboratory model glycerin-bath high temperature beaker-dyeing machine was used. The finely powdered dye $(20 \mathrm{mg})$ was intimately mixed with dispersing agent dodamol (40mg) in $100 \mathrm{ml}$ water. The mixture was then dispersed in an ultrasonic vibrator for 30 minutes at room temperature. The $\mathrm{pH}$ of the solution was adjusted 5.56.0 by adding acetic acid. The dyeing of the fabric was done at $130^{\circ} \mathrm{C}$ and $30 \mathrm{psi}$ pressure for 1 hour. These dyes provided colors in the range of red varying from bright red to pink with good levelness, brightness and depth on the fabric. Their dyeing properties are given in Table 2. The dyes were tested for light fastness and sublimation fastness. The light fastness test was done using a Microsal light fastness tester having Xenon vapour lamp. The dyed fabric was exposed to light along with the standard dye patterns of specific ratings of the grade 1-8. The sublimation fastness was assessed by keeping a composite specimen of dyed polyester between two undyed polyester pieces in a precision press at $200^{\circ} \mathrm{C}$ for 30 seconds. The change in color of the 
specimen was assessed with gray scales. The gray scale for the alteration of color consisted of grades 1-5. The pick-up values are based on standard depths, the pick-up values of the dyed polyester fibers varied from 2 to 3 , most of them had a pick-up value of 3 . The light fastness varied from grades 3 to 5 . The dyes $\mathbf{4 e}$ and $\mathbf{4 h}$ had poor light fastness, dyes $\mathbf{4 b}, \mathbf{4 c}$ and $\mathbf{4 g}$ showed a good light fastness of the grade 5. Dyes 4a, 4d, 4f and $\mathbf{4 i}$ had a fair light fastness of the grade 4. Dye 4c showed an excellent sublimation fastness (grade 5). Dyes $\mathbf{4 a}, \mathbf{4 b}, \mathbf{4 d}, \mathbf{4 e}, \mathbf{4 h}$ and 4i showed a very good sublimation fastness (grade 4). The dyes $\mathbf{4 f}$ and $\mathbf{4 g}$ had a good sublimation fastness of grade 3 . Thus most of these dyes showed very good dyeing properties.

Table 1. Characteristics of compounds $\mathbf{4 a - i}$ as dyes

\begin{tabular}{|c|c|c|c|c|c|c|}
\hline \multirow[t]{2}{*}{ Compound } & \multicolumn{4}{|c|}{ Substituent } & \multirow{2}{*}{$\begin{array}{l}\text { Absorption } \\
\text { maxima } \\
\lambda_{\max } \mathrm{nm} \\
(\mathrm{DMF})\end{array}$} & \multirow{2}{*}{$\begin{array}{c}\log \varepsilon \\
(\mathrm{DMF})\end{array}$} \\
\hline & $\mathrm{R}^{1}$ & $\mathrm{R}^{2}$ & $\mathrm{R}^{3}$ & $\mathrm{R}^{4}$ & & \\
\hline $4 a$ & $\mathrm{H}$ & $\mathrm{H}$ & $\mathrm{NHCOCH}_{3}$ & $\mathrm{H}$ & 523 & 4.60 \\
\hline $4 b$ & $\mathrm{OCOCH}_{3}$ & $\mathrm{OCOCH}_{3}$ & $\mathrm{NHCOCH}_{3}$ & $\mathrm{OCH}_{3}$ & 528 & 4.68 \\
\hline $4 c$ & $\mathrm{CN}$ & $\mathrm{H}$ & $\mathrm{CH}_{3}$ & $\mathrm{H}$ & 500 & 4.46 \\
\hline 4d & $\mathrm{CN}$ & $\mathrm{CN}$ & $\mathrm{H}$ & $\mathrm{H}$ & 502 & 4.65 \\
\hline $4 e$ & $\mathrm{OCOCH}_{3}$ & $\mathrm{OCOCH}_{3}$ & $\mathrm{Cl}$ & $\mathrm{H}$ & 480 & 4.50 \\
\hline 4f & $\mathrm{OCOCH}_{3}$ & $\mathrm{OCOCH}_{3}$ & $\mathrm{NHCOCH}_{3}$ & $\mathrm{H}$ & 525 & 4.63 \\
\hline $4 g$ & $\mathrm{OCOCH}_{3}$ & $\mathrm{OCOCH}_{3}$ & $\mathrm{H}$ & $\mathrm{H}$ & 505 & 4.71 \\
\hline $4 h$ & $\mathrm{OCOCH}_{3}$ & $\mathrm{CN}$ & $\mathrm{H}$ & $\mathrm{H}$ & 492 & 4.57 \\
\hline $4 i$ & $\mathrm{OCOCH}_{3}$ & $\mathrm{CN}$ & $\mathrm{NHCOCH}_{3}$ & $\mathrm{H}$ & 532 & 4.78 \\
\hline
\end{tabular}

Table 2. Dyeing properties of dyes 4a-i on polyester fabric

\begin{tabular}{clccc}
\hline Compound & $\begin{array}{l}\text { Color on dyed } \\
\text { polyester fabric }\end{array}$ & Pick up & Light fastness & $\begin{array}{c}\text { Sublimation } \\
\text { fastness }\end{array}$ \\
\hline $\mathbf{4 a}$ & Reddish violet & 3 & 4 & 4 \\
$\mathbf{4 b}$ & Very bright pink & 3 & 5 & 4 \\
$\mathbf{4 c}$ & Bright red & 3 & 5 & 5 \\
$\mathbf{4 d}$ & Reddish brown & 3 & 4 & 4 \\
$\mathbf{4 e}$ & Pink & 2 & 3 & 4 \\
$\mathbf{4 f}$ & Reddish brown & 3 & 4 & 3 \\
$\mathbf{4 g}$ & Bright red & 3 & 5 & 3 \\
$\mathbf{4 h}$ & Reddish violet & 3 & 3 & 4 \\
$\mathbf{4 i}$ & Very bright pink & 2 & 4 & 4 \\
\hline
\end{tabular}




\section{Experimental Section}

General Procedures. All the chemicals used in the synthesis of the thiazole $\mathbf{3}$ and dyes $\mathbf{4}$ were of commercial grade and were further purified by crystallization and distillation. The coupling components (N,N dialkylaniline derivatives) were sourced from Clariant limited. All solvents used were of analytical grade. The NMR spectra were recorded on a Varian $300 \mathrm{MHz} \mathrm{SW}$ multinuclear probe, I.R spectra were recorded on Perkin-Elmer Spectrum BX instrument. U.V spectrophotometer Analytik Jena Specord 50 was used for recording the absorption maxima of the dyes. Mass spectra were taken on LC-MS ThermoFinnigan navigator 30019 and PerkinElmer CHN analyzer 2400/series II was used for elemental analysis.

3,3,5-Trimethylcyclohexanone (2). 3,3,5-Trimethylcyclohexanone (2) was prepared as per reported literature ${ }^{30}$ by catalytic hydrogenation of isophorone (1).

2-Amino-5,5,7-trimethyl-4,5,6,7-tetrahydrobenzothiazole (3). Compound (2) (7 g, 0.05 mole) was dissolved in $35 \mathrm{ml}$ ethanol. Thiourea $(7.61 \mathrm{~g}, 0.1 \mathrm{~mole})$ and iodine $(12.7 \mathrm{~g}, 0.05$ mole $)$ were added and the reaction mixture was heated under reflux for $5 \mathrm{hrs}$. The reaction mixture was then cooled to $20^{\circ} \mathrm{C}$ and quenched in $200 \mathrm{ml}$ water. The quenched mass was basified with liquor ammonia solution and extracted in $200 \mathrm{ml}$ ethyl acetate. The ethyl acetate layer was washed with $100 \mathrm{ml}$ water and then filtered to remove insoluble solids. The ethyl acetate extract was then concentrated and the crude residue was purified by column chromatography using chloroform as eluent and silicagel (70-230 mesh) as solid phase. The pure fraction on concentration gave $6.4 \mathrm{~g}$ of pure 3 (yield $65 \%$ ). m.p: $55-57^{\circ} \mathrm{C}$, m.p of hydrochloride salt: $205^{\circ} \mathrm{C}$. NMR (300 MHz, $\left.\mathrm{CDCl}_{3}\right): \delta 0.95(2 \mathrm{H}, \mathrm{s}), 1.08(3 \mathrm{H}, \mathrm{d}), 1.18-1.24(6 \mathrm{H}, \mathrm{m}), 1.66(2 \mathrm{H}, \mathrm{m}), 2.75(1 \mathrm{H}, \mathrm{m}), 4.9(2 \mathrm{H}, \mathrm{b})$. I.R: $3392 \mathrm{~cm}^{-1}, 2953 \mathrm{~cm}^{-1}, 1362 \mathrm{~cm}^{-1}$. Mass spectra: $\mathrm{m} / \mathrm{z}=197[\mathrm{M}+\mathrm{H}]^{+}$. Calculated for $\mathrm{C}_{10} \mathrm{H}_{16} \mathrm{~N}_{2} \mathrm{~S}$ : \% C 61.22, H 8.16, N14.28 Found: C 61.46, H 8.33, N14.04.

Preparation of nitrosylsulfuric acid ${ }^{31}$. Concentrated sulfuric acid $(98 \%, 80 \mathrm{ml})$ was taken in a $250 \mathrm{ml}$ flask and cooled to $0^{\circ} \mathrm{C}$. Sodium nitrite $(6.9 \mathrm{~g}, 0.1 \mathrm{~mole})$ was added slowly into it under stirring maintaining temperature below $10^{\circ} \mathrm{C}$. After completion of the addition, stirring was continued further for 15 minutes. The reaction mixture was then gently heated to about $70^{\circ} \mathrm{C}$ and stirred at this temperature until all residual nitrite dissolved. The clear solution was then cooled to room temperature and used for diazotization.

2-[2-Acetylamino-4-( $N, N$-diethylamino)phenyl]azo-5,5,7-trimethyl-4,5,6,7-tetrahydrobenzothiazole (4a). Compound 3 (1.96 g, 0.01 mole) was dissolved with stirring in concentrated sulfuric acid $(98 \%, 5 \mathrm{ml})$. This solution was cooled to $0-10^{\circ} \mathrm{C}$. Ice-cold nitrosyl sulfuric acid $(10 \mathrm{ml})$ was added drop wise to the above solution over a period of 15 minutes. Stirring was continued at $0-10^{\circ} \mathrm{C}$ further for one hour. The solution was diluted with $5 \mathrm{ml}$ acetic acid. Excess nitrous acid was destroyed using urea $(0.15 \mathrm{~g}, 0.003 \mathrm{~mole})$.

The coupling component 3-Acetylamino- $N, N$-(diethyl)aniline (2.06 g, 0.01mole) was dissolved in acetic acid $(10 \mathrm{ml})$. The solution was cooled to $0-5^{\circ} \mathrm{C}$. The diazo solution prepared above was slowly added into the coupler solution with vigorous stirring over a period of 15 minutes at 0 - 
$5^{\circ} \mathrm{C}$. Stirring was continued further at $0-5^{\circ} \mathrm{C}$ for $2 \mathrm{hrs}$. The $\mathrm{pH}$ of reaction mass was adjusted to 4-5 by addition of saturated sodium acetate solution and stirred at room temperature for 2-3 hrs. The dye was precipitated at $\mathrm{pH} 6$ by addition of $10 \%$ Sodium carbonate solution. The dye was filtered and washed with water till acid free and dried at $50^{\circ} \mathrm{c}$. The crude dye was purified by column chromatography using chloroform as eluent and silicagel (70-230 mesh) as solid phase. Yield: $3.1 \mathrm{~g}(75 \%)$. m.p: $136^{\circ} \mathrm{C} .{ }^{1} \mathrm{H}$ NMR $\left(300 \mathrm{MHz}, \mathrm{CDCl}_{3}\right): \delta 1.0(2 \mathrm{H}, \mathrm{s}), 1.13(3 \mathrm{H}, \mathrm{d}), 1.3-1.4$ $(12 \mathrm{H}, \mathrm{m}), 1.6(2 \mathrm{H}, \mathrm{m}), 2.29(3 \mathrm{H}, \mathrm{s}), 2.97(1 \mathrm{H}, \mathrm{m}), 3.5(4 \mathrm{H}, \mathrm{qt}), 6.5(1 \mathrm{H}$, aromatic $), 7.7(1 \mathrm{H}$, aromatic), $8.07\left(1 \mathrm{H}\right.$, aromatic). I.R: $3436 \mathrm{~cm}^{-1}, 2957 \mathrm{~cm}^{-1}, 1512 \mathrm{~cm}^{-1}, 824 \mathrm{~cm}^{-1}$. Mass spectra: $\mathrm{m} / \mathrm{z}=414[\mathrm{M}+\mathrm{H}]^{+}$. Calculated for $\mathrm{C}_{22} \mathrm{H}_{31} \mathrm{~N}_{5} \mathrm{OS}: \% \mathrm{C}$ 63.92, H 7.50, N16.95 Found: C 63.90, H 7.46, N16.88

2-[2-Acetylamino-5-methoxy-4-\{N,N-bis(2-acetoxyethyl)amino\}phenyl]azo-5,5,7-trimethyl4,5,6,7-tetrahydrobenzothiazole (4b). Compound (4b) was synthesized using the above procedure with 5-acetylamino-2-methoxy-1- $\mathrm{N}, \mathrm{N}$-bis(2-acetoxy ethyl)aniline as coupling component. Yield: $4.3 \mathrm{~g}(77 \%)$. m.p: $253^{\circ} \mathrm{C} .{ }^{1} \mathrm{H}$ NMR $\left(\mathrm{CDCl}_{3}\right): \delta 0.99(2 \mathrm{H}, \mathrm{s}), 1.14(3 \mathrm{H}, \mathrm{d}), 1.35$ $(6 \mathrm{H}, \mathrm{m}), 1.59(2 \mathrm{H}, \mathrm{m}), 2.04(6 \mathrm{H}, \mathrm{s}), 2.27(3 \mathrm{H}, \mathrm{s}), 3.0(1 \mathrm{H}, \mathrm{m}), 3.7(4 \mathrm{H}, \mathrm{t}), 3.82(3 \mathrm{H}, \mathrm{s}), 4.29(3 \mathrm{H}$, t), $7.39\left(1 \mathrm{H}\right.$, aromatic), $8.25\left(1 \mathrm{H}\right.$, aromatic), $9.4(1 \mathrm{H}, \mathrm{b})$. I.R: $3437 \mathrm{~cm}^{-1}, 2926 \mathrm{~cm}^{-1}, 1502 \mathrm{~cm}^{-1}$, $815 \mathrm{~cm}^{-1}$. Mass spectra: $\mathrm{m} / \mathrm{z}=560[\mathrm{M}+\mathrm{H}]^{+}$. Calculated for $\mathrm{C}_{27} \mathrm{H}_{37} \mathrm{~N}_{5} \mathrm{O}_{6} \mathrm{~S}: \% \mathrm{C} 57.96, \mathrm{H} 6.62, \mathrm{~N}$ 12.52 Found: C 57.70, H 6.56, N 12.56.

2-[2-Methyl-4-\{( $N$-ethyl, $N$-cyanoethyl)amino\}phenyl]azo-5,5,7-trimethyl-4,5,6,7-tetrahydrobenzothiazole (4c). Compound (4c) was synthesized using the above procedure with $N$-ethyl$\mathrm{N}$-(2-cyanoethyl)-3-toludine as coupling component. Yield: $2.88 \mathrm{~g}$ (73\%). m.p: $123^{\circ} \mathrm{C} .{ }^{1} \mathrm{H}$ NMR $\left(\mathrm{CDCl}_{3}\right): \delta 0.98(2 \mathrm{H}, \mathrm{s}), 1.13(3 \mathrm{H}, \mathrm{d}), 1.24-1.36(9 \mathrm{H}, \mathrm{m}), 1.63(2 \mathrm{H}, \mathrm{m}), 2.61-2.69(5 \mathrm{H}, \mathrm{m}), 3.0$ $(1 \mathrm{H}, \mathrm{m}), 3.5(2 \mathrm{H}, \mathrm{qt}), 3.75(2 \mathrm{H}, \mathrm{t}), 6.54\left(2 \mathrm{H}\right.$, aromatic), $7.98\left(1 \mathrm{H}\right.$, aromatic). I.R: $2956 \mathrm{~cm}^{-1}$, $1531 \mathrm{~cm}^{-1}, 797 \mathrm{~cm}^{-1}$. Mass spectra: $\mathrm{m} / \mathrm{z}=396[\mathrm{M}+\mathrm{H}]^{+}$. Calculated for $\mathrm{C}_{22} \mathrm{H}_{29} \mathrm{~N}_{5} \mathrm{~S}: \% \mathrm{C} 66.83, \mathrm{H}$ 7.34, N 17.72 Found: C 66.98, H 7.54, N 17.52.

2-[4-\{ $N, N$-Bis(2-cyanoethyl)amino\}phenyl]azo-5,5,7-trimethyl-4,5,6,7-tetrahydrobenzothiazole (4d). Compound (4d) was synthesized using the above procedure with $N, N$-bis(2cyanoethyl)aniline as coupling component. Yield: $3.12 \mathrm{~g}(77 \%)$ m.p: $119^{\circ} \mathrm{C} .{ }^{1} \mathrm{H}$ NMR $\left(\mathrm{CDCl}_{3}\right)$ : $\delta 0.99(2 \mathrm{H}, \mathrm{s}), 1.14(3 \mathrm{H}, \mathrm{d}), 1.32(6 \mathrm{H}, \mathrm{m}), 1.60(2 \mathrm{H}, \mathrm{m}), 2.98(1 \mathrm{H}, \mathrm{m}), 3.5(4 \mathrm{H}, \mathrm{t}), 3.70(4 \mathrm{H}, \mathrm{t})$, $6.61\left(2 \mathrm{H}\right.$, aromatic), $7.83\left(2 \mathrm{H}\right.$, aromatic) I.R: $2958 \mathrm{~cm}^{-1}, 2124 \mathrm{~cm}^{-1}, 1522 \mathrm{~cm}^{-1}, 806 \mathrm{~cm}^{-1}$. Mass spectra: $\mathrm{m} / \mathrm{z}=407[\mathrm{M}+\mathrm{H}]^{+}$. Calculated for $\mathrm{C}_{22} \mathrm{H}_{26} \mathrm{~N}_{6} \mathrm{~S}$ : \% C 65.02, H 6.40, N 20.69 Found: $\mathrm{C}$ 65.30, H 6.16, N 20.45

2-[2-Chloro-4-\{N,N-bis(2-acetoxyethyl)amino\}phenyl]azo-5,5,7-trimethyl-4,5,6,7-tetrahydrobenzothiazole (4e). Compound (4e) was synthesized using the above procedure with 3-chloro$N, N$-bis(2-acetoxyethyl)aniline as coupling component. Yield: $3.8 \mathrm{~g}(75 \%)$ m.p: $266^{\circ} \mathrm{C} .{ }^{1} \mathrm{H}$ NMR $\left(\mathrm{CDCl}_{3}\right): \delta 0.98(2 \mathrm{H}, \mathrm{s}), 1.12(3 \mathrm{H}, \mathrm{d}), 1.33(6 \mathrm{H}, \mathrm{m}), 1.60(2 \mathrm{H}, \mathrm{m}), 2.04(6 \mathrm{H}, \mathrm{s}), 3.0(1 \mathrm{H}$, $\mathrm{m}), 3.6(4 \mathrm{H}, \mathrm{t}), 4.33(3 \mathrm{H}, \mathrm{t}), 6.53\left(2 \mathrm{H}\right.$, aromatic), $7.78\left(1 \mathrm{H}\right.$, aromatic). I.R: $2926 \mathrm{~cm}^{-1}, 1739 \mathrm{~cm}^{-1}$, $1529 \mathrm{~cm}^{-1}, 831 \mathrm{~cm}^{-1}$. Mass spectra: $\mathrm{m} / \mathrm{z}=508[\mathrm{M}+\mathrm{H}]^{+}$. Calculated for $\mathrm{C}_{24} \mathrm{H}_{31} \mathrm{ClN}_{4} \mathrm{O}_{4} \mathrm{~S}: \% \mathrm{C}$ 56.86, H 6.12, N 11.05 Found: C 56.64, H 6.06, N 11.22. 
2-[2-Acetylamino-4-\{N,N-bis(2-acetoxyethyl)amino\}phenyl]azo-5,5,7-trimethyl-4,5,6,7tetrahydrobenzothiazole (4f). Compound (4f) was synthesized using the above procedure with 3- $N, N$-bis(2-acetoxyethyl)amino acetanilide as coupling component. Yield: $3.97 \mathrm{~g}$ (75\%). m.p: $238^{\circ} \mathrm{C} .{ }^{1} \mathrm{H}$ NMR $\left(\mathrm{CDCl}_{3}\right): \delta 1.0(2 \mathrm{H}, \mathrm{s}), 1.13(3 \mathrm{H}, \mathrm{d}), 1.35(6 \mathrm{H}, \mathrm{m}), 1.60(2 \mathrm{H}, \mathrm{m}), 2.04(6 \mathrm{H}, \mathrm{s})$, $2.18(3 \mathrm{H}, \mathrm{s}), 3.0(1 \mathrm{H}, \mathrm{m}), 3.65(4 \mathrm{H}, \mathrm{t}), 4.28(3 \mathrm{H}, \mathrm{t}), 6.55(2 \mathrm{H}$, aromatic $), 7.8(1 \mathrm{H}$, aromatic). I.R: $3413 \mathrm{~cm}^{-1}, 2959 \mathrm{~cm}^{-1}, 1739 \mathrm{~cm}^{-1}, 1529 \mathrm{~cm}^{-1}, 744 \mathrm{~cm}^{-1}$. Mass spectra: $\mathrm{m} / \mathrm{z}=530[\mathrm{M}+\mathrm{H}]^{+}$. Calculated for $\mathrm{C}_{26} \mathrm{H}_{35} \mathrm{~N}_{5} \mathrm{O}_{5} \mathrm{~S}: \%$ C 58.98, H 6.61, N 13.23 Found: C 59.22, H 6.56, N 12.96.

2-[4-\{N,N-Bis(acetoxyethyl)amino\}phenyl]azo-5,5,7-trimethyl-4,5,6,7-tetrahydrobenzo-

thiazole (4g). Compound (4g) was synthesized using the above procedure with $N, N$-bis(2acetoxyethyl)aniline as coupling component. Yield: $3.41 \mathrm{~g}(72 \%)$. m.p: $203^{\circ} \mathrm{C} .{ }^{1} \mathrm{H}$ NMR $\left(\mathrm{CDCl}_{3}\right): \delta 1.0(2 \mathrm{H}, \mathrm{s}), 1.13(3 \mathrm{H}, \mathrm{d}), 1.33(6 \mathrm{H}, \mathrm{m}), 1.58(2 \mathrm{H}, \mathrm{m}), 2.05(6 \mathrm{H}, \mathrm{s}), 3.0(1 \mathrm{H}, \mathrm{m}), 3.63$ $(4 \mathrm{H}, \mathrm{t}), 4.32(3 \mathrm{H}, \mathrm{t}), 6.6\left(2 \mathrm{H}\right.$, aromatic), $7.75\left(2 \mathrm{H}\right.$, aromatic). I.R: $2956 \mathrm{~cm}^{-1}, 1698 \mathrm{~cm}^{-1}, 1531$ $\mathrm{cm}^{-1}, 751 \mathrm{~cm}^{-1}$ Mass spectra: $\mathrm{m} / \mathrm{z}=473[\mathrm{M}+\mathrm{H}]^{+}$. Calculated for $\mathrm{C}_{24} \mathrm{H}_{32} \mathrm{~N}_{4} \mathrm{O}_{4} \mathrm{~S}: \% \mathrm{C} 61.07, \mathrm{H}$ 6.78, N 11.86 Found: C 60.86, H 6.94, N 11.58.

2-[4-\{N-(2-Acetoxyethyl)- $N$-(2-cyanoethyl)amino\}phenyl]azo-5,5,7-trimethyl-4,5,6,7-tetrahydrobenzothiazole (4h). Compound (4h) was synthesized using the above procedure with $N$ (2-acetoxyethyl)- $N$-(2-cyanoethyl)aniline as coupling component. Yield: 3.29 g (75\%). m.p: $211^{\circ} \mathrm{C} .{ }^{1} \mathrm{H}$ NMR $\left(\mathrm{CDCl}_{3}\right): \delta 1.0(2 \mathrm{H}, \mathrm{s}), 1.13(3 \mathrm{H}, \mathrm{d}), 1.33(6 \mathrm{H}, \mathrm{m}), 1.58(2 \mathrm{H}, \mathrm{m}), 2.05(3 \mathrm{H}, \mathrm{s})$, $2.6(4 \mathrm{H}, \mathrm{t}), 3.0(1 \mathrm{H}, \mathrm{m}), 3.85(2 \mathrm{H}, \mathrm{t}), 4.23(2 \mathrm{H}, \mathrm{t}), 6.58(2 \mathrm{H}$, aromatic $), 7.8(2 \mathrm{H}$, aromatic). I.R: $2958 \mathrm{~cm}^{-1}, 1741 \mathrm{~cm}^{-1}, 1522 \mathrm{~cm}^{-1}, 806 \mathrm{~cm}^{-1}$. Mass spectra: $\mathrm{m} / \mathrm{z}=440[\mathrm{M}+\mathrm{H}]^{+}$. Calculated for $\mathrm{C}_{23} \mathrm{H}_{29} \mathrm{~N}_{5} \mathrm{O}_{2} \mathrm{~S}: \%$ C 62.87, H 6.60, N 15.94 Found: C 62.70, H 6.76, N 15.72.

2-[2-Acetylamino-4- $\{N$-(2-acetoxyethyl)- $N$-(2-cyanoethyl)amino\}phenyl]azo-5,5,7-trimethyl -4,5,6,7-tetrahydrobenzothiazole (4i). Compound (4i) was synthesized using the above procedure with 3-acetylamino[ $N$-(2-acetoxyethyl)- $N$-(2-cyanoethyl)]aniline as coupling component. Yield: $3.62 \mathrm{~g}(73 \%)$. m.p: $181^{\circ} \mathrm{C} .{ }^{1} \mathrm{H}$ NMR $\left(\mathrm{CDCl}_{3}\right): \delta 1.0(2 \mathrm{H}, \mathrm{s}), 1.13(3 \mathrm{H}, \mathrm{d}), 1.33$ $(6 \mathrm{H}, \mathrm{m}), 1.58(2 \mathrm{H}, \mathrm{m}), 2.05(3 \mathrm{H}, \mathrm{s}), 2.33(3 \mathrm{H}, \mathrm{s}), 2.72(4 \mathrm{H}, \mathrm{t}), 2.96(1 \mathrm{H}, \mathrm{m}), 3.8(2 \mathrm{H}, \mathrm{t}), 4.28$ $(2 \mathrm{H}, \mathrm{t}), 6.62\left(2 \mathrm{H}\right.$, aromatic), $7.85\left(1 \mathrm{H}\right.$, aromatic), $\delta 9.45(1 \mathrm{H}, \mathrm{b})$. I.R: $3400 \mathrm{~cm}^{-1}, 2957 \mathrm{~cm}^{-1}$, $1738 \mathrm{~cm}^{-1}, 1515 \mathrm{~cm}^{-1}, 827 \mathrm{~cm}^{-1}$ Mass spectra: $\mathrm{m} / \mathrm{z}=497[\mathrm{M}+\mathrm{H}]^{+}$. Calculated for $\mathrm{C}_{25} \mathrm{H}_{32} \mathrm{~N}_{6} \mathrm{O}_{3} \mathrm{~S}$ : \% C 60.48, H 6.45, N 16.94 Found: C 60.72, H 6.58, N 16.66.

\section{Acknowledgements}

We would like to thank Dr.S.B.Bhirud of Glenmark Pharmaceuticals ltd. for his support to carry out this work. We also thank Prof. V. Kanetkar of Dyes department, UICT, Mumbai for the help provided for testing of the dyes. 


\section{References}

1. Shuttleworth, L.; Weaver, M. A. The chemistry and application of Dyes; Plenum Press: New York, 1970; p 107.

2. Mitsubishi Chemical industries Co. Ltd., Jap.Patent 1980, 55005966; Chem. Abstr. 1980, 92,199750x.

3. ICI Ltd., Ger. Offen. 1977, 2,633,910; Chem. Abstr.1977, 86, 157035.

4. Tappe, H.; Roth, K.; Cassella, A. G.; Ger. Offen. 3,036578; Chem. Abstr 1982, 97, 571167z.

5. Weaver, M. A.; Priagen, H. S.; Eastman Kodak Co. US Patent 1972, 36,39,385; Chem. Abstr. 1972, 76, 15583k.

6. Sartori, M. A.; E.I Du Pont De Nemours and Co. US Patent 1967, 3,336,286; Chem. Abstr. 1967, 67, 100999j.

7. Ponnuswami, J.; Ciba Geigy Ger. Offen. 1976, 2,613,595; Chem. Abstr.1977, 86, 44750z.

8. Mitsui Toatsu Chemical Inc. Jap. Patent 1979, 540,927,071; Chem. Abstr.1979, 91, 75697v.

9. Nippon Kayaku Co. 1td. Jap. Patent 1986, 6128555; Chem. Abstr.1986, 105, 135408w.

10. Towne, E. B.; Hill, M.H. US Patent 1956, 2,746,953; Chem. Abstr.1956, 50, 6058.

11. Merian, E. US Patent 1961, 3007915; Chem. Abstr.1962, 57, 7421.

12. Egli, R. US Patent 1983, 4395544; Chem. Abstr.1983, 99, 159949.

13. Towne, E. B.; Dickey, J. B.; Bloom, M. S. US Patent 1958, 2852504; Chem. Abstr. 1959, 53, 6627.

14. Straley, J.; Wallance, D.; Eastman Kodak Co. US Patent 1965, 3206452; Chem. Abstr. 1965, $63,1831 \mathrm{~h}$.

15. Weaver, M. A.; Wallance, D. J.; Eastman Kodak Co. US Patent 1974, 3787388; Chem. Abstr.1974, 80, 122395e.

16. Venkatraman, K. The Chemistry of Synthetic dyes, Academic Press: New York, 1952; Vol. 2, p 1003.

17. Dickey, J. B.; Towne, E. B.; Eastman Kodak Co. US Patent 1955, 2659719; Chem. Abstr. 1955, 49, 1335.

18. Gosei Senryo; Gijupsu Kenktu Kumaiai. Jap. Patent 1983, 59215355; Chem. Abstr. 1983, 102, 186655.

19. Tsein. H; Chu. K. P; Chang. H. US Patent 1997, 5652344; Chem. Abstr. 1997, 127, 150201.

20. Bello, K. A.; Martins, C. M. O. A.; Adamu, I. K. Colorage 1995, 42, 29.

21. Desai, K. R.; Modi, B. R.; Mistry, B. D. J. Indian Chem. Soc. 1995, 72, 141.

22. Bello, K. A. Dyes Pigm. 1995, 27, 45.

23. Hallas, G.; Choi, J. H. Dyes Pigm. 1999, 42, 249.

24. Chikhalia, K. H.; Desai, K. R. J. Inst. Chem. (India) 1998, 70, 142.

25. Rangnekar, D. W.; Kanetkar, V. R.; Shankarling, G. S.; Malanker, J. V. J. Indian Counc. Chem. 1998, 15, 28.

26. Towns, A. D. Dyes Pigm. 1999, 42, 3.

27. Hantzsch, A. R.; Weber, J. H. Ber. 1887, 20, 3118. 
28. Dodson, R. M.; Carroll King, L. J. Am. Chem. Soc. 1944, 67, 2242.

29. Carroll King, L.; Hlavacek Robert, J. J. Am. Chem. Soc. 1950, 72, 3722.

30. Uehara, R.; Iwatani. Masayoshi, Nagahashi. Takao Jap. Patent 1970, 51024497; Chem. Abstr.1977, 86, 43290.

31. Euard, H.; Blangey, L. Fundamental process of dye chemistry, Fierz-David, 1949, p 247. 